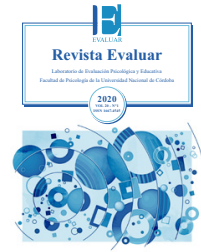

\title{
Propiedades psicométricas de una Escala de Phubbing en una muestra argentina
}

\author{
Psychometric properties of a Phubbing Scale in an Argentinean sample
}

\author{
Santiago Resett * 1 , Pablo Christian González-Caino ${ }^{2}$
}

Introducción Objetivos Metodología Resultados Discusión Referencias

1- Universidad Argentina de la Empresa (UADE). Instituto de Ciencias Sociales y Disciplinas Proyectuales (INSOD), Buenos Aires, Argentina. 2- CONICET. Buenos Aires, Argentina.

Recibido: 19/05/2020 Revisado: 11/06/2020 Aceptado: 17/06/2020

\section{Resumen}

El phubbing es una conducta de gran actualidad escasamente estudiada en la Argentina. La presente investigación tuvo como objetivo adaptar un cuestionario a este respecto en una muestra de adolescentes y adultos emergentes/jóvenes argentinos. Se constituyó una muestra intencional de 1245 participantes de la Ciudad Autónoma de Buenos Aires y Paraná, Argentina (edades de 15 a 40 años, $70 \%$ mujeres). Los participantes contestaron la Escala de Phubbing de Karadağ et al. (2015) y la Subescala de Conflicto-Antagonismo con progenitores (padres y madres) del Inventario de Furman y Buhrmester (1992). Los resultados de un análisis paralelo indicaron una estructura adecuada de tres factores. Un análisis factorial confirmatorio mostró un ajuste satisfactorio para dicho modelo. La confiabilidad interna se consideró adecuada con ciertas reservas para algunos de sus factores. Se encontraron evidencias de validez de constructo para la escala: la asociación del phubbing con la edad y con el conflicto-antagonismo con progenitores.

Palabras clave: phubbing, celulares, escala, propiedades, psicometría

\begin{abstract}
Phubbing is a behavior of great relevance, yet scarcely studied in Argentina. The present study validated a questionnaire in this regard in a sample of Argentine adolescents and emerging adults/young adults. An intentional sample of 1245 participants from the Autonomous City of Buenos Aires and Paraná, Argentina, aged 12 to 40 years, with $70 \%$ of women was constituted. Participants answered the Karadağ et al. (2015) Questionnaire on Phubbing and the Furman and Buhrmester (1992) Inventory of Conflict-Antagonism with parents (fathers and mothers). The results of exploratory factor analysis indicated an adequate structure of three factors. Confirmatory factor analysis showed a satisfactory adjustment. Internal reliability was adequate with certain reservations for some factors. Construct validity evidence was also found, as indicated by the association of phubbing with age and conflict-antagonism with parents.
\end{abstract}

Key words: phubbing, mobile phones, scale, properties, psychometrics

\footnotetext{
* Correspondencia a: Santiago Resett, Laurencena 222 bis, Paraná, Entre Ríos, Argentina, CP 3100. Teléfono: 549343 6101147. E-mail: santiago_resett@hotmail.com
}

Cómo citar este artículo: Resett, S., \& González-Caino, P. C. (2020). Propiedades psicómetricas de una Escala de Phubbing en una muestra argentina. Revista Evaluar, 20(2), 69-84. Recuperado de https://revistas.unc.edu.ar/index.php/revaluar 


\section{Introducción}

La tecnología ha alcanzado un crecimiento sin precedentes en las últimas décadas. Así, el uso de las nuevas tecnologías de la información y la comunicación (TIC) — como celulares y computadoras - se ha desarrollado notablemente, y ha adquirido una gran popularidad en los adolescentes y adultos emergentes. En los Estados Unidos, por ejemplo, el 92\% de los adolescentes entre las edades 13-17 señalaba usar celulares y un $25 \%$ afirmaba hacerlo todo el tiempo (Lenhart, 2015). En los países latinoamericanos, como la Argentina, la disponibilidad y la atracción hacia las nuevas tecnologías son notables, con los adolescentes a la vanguardia de la generación interactiva debido al alto uso de las TIC (Facio \& Resett, 2012). Un ejemplo de ello es que en el año 2019 el 21\% de los adolescentes argentinos señalaba tener computadora en su propia habitación, el 97\% poseía celular y un $78 \%$ contaba con acceso a la Internet, según el Instituto Nacional de Estadísticas y Censos (2019).

El celular es la tecnología que más rápido se ha desarrollado en la historia de la humanidad, según la Unión Internacional de Telecomunicaciones (2013). De este modo, los celulares son utilizados cada vez más en distintos ámbitos para facilitar la conexión entre las personas y solucionar distintos problemas de su vida cotidiana. Sin embargo, el despliegue de las TIC también ha acarreado nuevos problemas, como los conocidos fenómenos del trolling, el cyberbullying, el grooming o el sexting (Resett \& González-Caino, 2019). Otra conducta negativa que se incrementó en el último tiempo es el phubbing. Sin embargo, este ha sido menos estudiado que las conductas antes mencionadas.

El phubbing se compone de las palabras en inglés phone (teléfono) y snubbing (despreciar). Dicho constructo se caracteriza por ser una con- ducta por la cual un individuo ignora a otro al estar utilizando el celular u otro dispositivo similar, como una tablet (Abramova, Baumann, Krasnova, \& Lessmann, 2017; Nazir \& Pişkin, 2016; Ugur \& Koc, 2015; Vanden-Abeele, Antheunis, \& Schouten, 2016). Con respecto al origen del término, este se remonta al año 2012 y se atribuye a la agencia de publicidad McCann de Melbourne, Australia (Capilla-Garrido \& Cubo-Delgado, 2017).

Con el crecimiento de la duración de las baterías de los celulares y la posibilidad de estar conectado a la Internet todo el tiempo, la conducta de phubbing se genera con mayor frecuencia y en diferentes entornos, como las reuniones de amigos. Si bien es un fenómeno que se da en todas las edades, es más habitual en los adolescentes y los jóvenes.

El phubbing se considera un constructo multidimensional. Por ejemplo, Karadağ et al. (2015) indican que puede explicarse por la adicción al teléfono móvil, a mandar mensajes de texto en forma reiterada, a las redes sociales, a la Internet y a los videojuegos. Chotpitayasunondh y Douglas (2016) detectaron correlaciones moderadas entre el comportamiento de phubbing y la adicción a los teléfonos inteligentes e Internet. Así, un phubber - la persona que lleva a cabo dicho comportamiento - puede experimentar incapacidad para monitorear o controlar la conducta que lleva a cabo con su teléfono inteligente, dificultad para regular su uso y mayor compulsión por el empleo de las TIC (Chotpitayasunondh \& Douglas, 2016). Aunque el phubbing puede aparentar ser una conducta menor, los costos psicosociales de la adicción a los celulares están bien establecidos, y entre ellos se cuentan problemas de concentración (Hiscock, 2004) y problemas emocionales (Hong, Chiu, \& Huang, 2012). Incluso, un mal uso de dichos dispositivos puede poner en riesgo la vida, como en el caso de usar el celu- 
lar mientras se está conduciendo (Walsh, White, Hyde, \& Watson, 2008). También se ha comprobado que el phubbing está estrechamente relacionado con el ambiente familiar de los sujetos (Liu et al., 2019). Muchas investigaciones detectaron que el uso excesivo de TIC se asociaba con un deterioro en los vínculos entre progenitores e hijos adolescentes (Huang, Hu, Ni, Qin, \& Lü, 2019; Stockdale, Coyne, \& Padilla-Walker, 2018). Sin embargo, según nuestro conocimiento, no existen estudios que hayan evaluado directamente la involucración del phubbing en la calidad de las relaciones con los progenitores. Karadağ et al. (2016) afirman que el phubbing puede afectar las relaciones interpersonales y dañar las habilidades para la comunicación.

Si un aspecto central del phubbing es la adicción a los celulares, es probable que altos niveles de esta conducta aumenten los intercambios negativos con madres y padres. Más aún si se tiene en cuenta que la familia es una organización de gran relevancia psicosocial para los individuos y que en la Argentina los valores familistas son elevados. En dicho país - perteneciente a la tradición cultural latina - numerosas investigaciones con adolescentes y adultos emergentes hallaron elevados niveles de valores familiares (Facio, Resett, Micocci, \& Mistrorigo, 2007; Facio, Resett, Mistrorigo, \& Micocci, 2006). El phubbing puede ser nocivo en cualquier cultura, pero - principalmente- en un país en donde los valores familiares son elevados.

Debido a la importancia psicosocial del phubbing, su frecuencia y su escaso estudio, es preponderante la necesidad de desarrollar y adaptar instrumentos con sólidas bondades psicométricas para su medición y detección. Entre los cuestionarios que existen a este respecto, la Escala de Phubbing de Karadağ et al. (2015), desarrollada en Turquía, es una de las pocas existentes para evaluar dicha conducta. Se ha encontrado para ella sólida evidencia respecto a sus propiedades psicométricas, como confiabilidad interna y validez de constructo (Karadağ et al., 2015). En España también se ha obtenido evidencia de adecuadas propiedades psicométricas (Blanca \& Bendayan, 2018). En sí, se puede establecer que las ventajas de dicha escala son sus bondades psicométricas y el hecho de que solamente consta de 10 preguntas. Por otra parte, las ventajas de los autoinformes radican en que son técnicas de fácil aplicación, interpretación, con bajos costos económicos y con posibilidad de aplicarse en múltiples ocasiones para observar la estabilidad temporal de una variable (Hartung, Little, Allen, \& Page, 2011).

A pesar de la relevancia de la temática en nuestro país, no existen estudios que hayan evaluado las propiedades de un instrumento para medir el phubbing. De este modo, el objetivo de la presente investigación fue evaluar las propiedades psicométricas de la Escala de Phubbing de Karadağ et al. (2015) en una muestra de adolescentes y adultos emergentes/jóvenes de la Argentina.

\section{Objetivos}

- Evaluar la estructura factorial de la Escala de Phubbing en una muestra argentina.

- Determinar su consistencia interna.

- Explorar su validez concurrente con la edad y el conflicto-antagonismo percibido con los progenitores.

\section{Metodología}

Muestra

Se constituyó una muestra intencional de 1245 adolescentes, adultos emergentes y adultos jóvenes de la Ciudad Autónoma de Buenos Aires 
y de Paraná, Entre Ríos, Argentina, con edades de 15 a 40 años $\left(M_{\text {edad }}=21.07 ; \mathrm{DE}=4.74\right)$. Un $20 \%$ eran adolescentes (15-17 años), un 68\%, adultos emergentes (18-29 años) y el grupo restante, adultos jóvenes (30-40 años). Dicha agrupación en edad está altamente aceptada en el mundo y es la sugerida por Arnett (2013), quien es creador del término adultez emergente. Se decidió escoger estos grupos de edad debido a que el phubbing está muy extendido en ellos, principalmente en los adultos emergentes y jóvenes (Liu et al., 2019). Por otra parte, dicha población generalmente tiene todavía un contacto con sus progenitores e - incluso - la vasta mayoría reside con ellos, como se observó en el presente estudio. Con respecto al género, un $73 \%$ eran mujeres. Un $64 \%$ señaló que residía con ambos progenitores y el $72 \%$ de los participantes se percibía de clase socio-económica media. Con respecto al uso del celular, un 6\% dijo usarlo nada o casi nada; 30\%, 2-3 horas; $31 \%$, 4-8 horas y 33\%, más de 8 horas. Un grupo menor de 23 participantes fue excluido de la investigación al no contestar las preguntas de conflicto-antagonismo por tener sus padres fallecidos o no tener contacto con ellos.

\section{Instrumentos}

Cuestionario sociodemográfico. Se utilizó un cuestionario donde se solicitaban datos de género, edad, convivientes, clase socio-económica, nivel de escolaridad, cantidad de horas de uso del celular y fecha de la encuesta.

\section{Escala de Phubbing de Karadă̆ et al. (2015).}

Es una escala de 10 ítems con cinco alternativas Likert, que van de 1 (nunca) a 5 (siempre). Ejemplo de estos ítems puede ser: Cuando estoy con mi familia, siempre estoy ocupado con mi celular. Un análisis factorial exploratorio con rotación oblimin mostró dos factores para dicha escala: alteración de la comunicación y obsesión por el celular (Karadağ et al., 2015). Las primeras cinco preguntas conforman el primer factor $y$ las restantes, el segundo. El instrumento se adaptó al español argentino utilizando un método de traducción inversa siguiendo recomendaciones internacionales (International Test Commission, 2017;Muñiz, Elosua, \& Hambleton, 2013). Dos traductores profesionales en inglés tradujeron el original al español, así, junto con los autores del presente trabajo calificaron la equivalencia de las dos versiones y revisaron sistemáticamente cada uno de los ítems hasta llegar a un consenso. También se usó como referencia la versión en español de España de Blanca y Bendayan (2018). Luego, la versión alcanzada fue traducida nuevamente al inglés por un traductor diferente (un hablante nativo de inglés con conocimiento en español). El equipo de investigación comparó la versión original en inglés y la traducida para garantizar la equivalencia, tanto semántica como conceptual. Finalmente, se administró a 50 estudiantes universitarios en psicología en una sesión piloto. Al igual que en otros estudios (por ejemplo, Sánchez-Rosas, 2015), en dicha sesión se buscó explorar los significados que los participantes le brindaban a los términos. No emergieron dudas y para todos los participantes el lenguaje era claro, por lo que no tuvieron dificultades para responder. En dicha muestra piloto se halló un coeficiente alfa de Cronbach de .74 y .69 para las dos dimensiones respectivas propuestas por los autores del test. La única diferencia que presenta esta versión argentina con la original es que el ítem cinco (No creo que moleste a mi pareja cuando estoy ocupado con mi teléfono móvil) se reformuló como una declaración positiva, al igual que en la versión española de Blanca y Bendayan (2018). Este ítem ha presentado problemas de comprensión debido a la dirección negativa de la oración, 
dado que todos los demás elementos se presentan como declaraciones positivas. La investigación ha demostrado que es preferible un instrumento con todos los elementos formulados en la misma dirección (Solís-Salazar, 2015; Suárez-Alvarez et al., 2018; Van Sonderen, Sanderman, \& Coyne, 2013). También se agregó la opción amigos en la formulación del mismo ítem para evitar sesgos de respuesta o que solamente contestaran dicha pregunta quienes tenían pareja amorosa.

\section{Subescala Conflicto-Antagonismo con progeni-} tores del Inventario Red de Relaciones de Furman y Buhrmester (1992). Este inventario evalúa las percepciones que niños y jóvenes tienen de las relaciones con sus otros significativos en función de 10 cualidades relacionales. Aquí se lo empleó para evaluar la calidad de la relación con progenitores (madre y padre). Cada escala está constituida por tres preguntas de cinco alternativas cada una, que van desde (1) poco o nada hasta (5) al máximo. En esta encuesta se incluyó solamente la subescala de conflicto y antagonismo. Se les pedía a los sujetos evaluar los antagonismos en el vínculo con madre y padre. Se derivó un índice de conflicto-antagonismo debido a que las mismas son dos subescalas de altísima correlación entre sí en la Argentina (Facio et al., 2006). Las propiedades psicométricas de dicho instrumento han sido sólidamente establecidas en numerosos estudios nacionales con muestras de adolescentes y adultos emergentes (Facio et al., 2006; Facio \& Resett, 2014). El coeficiente alfa de Cronbach para la presente muestra fue .89 . Se escogió la percepción del conflicto y antagonismo con progenitores para evaluar la validez de constructo de la Escala de Phubbing, debido a que esta variable puede afectar las relaciones interpersonales, y por el alto nivel de familismo que se ha detectado en la Argentina, como se señaló previamente.

\section{Procedimiento de recolección de datos}

La recolección de datos fue realizada durante los meses de marzo a setiembre del año 2019 en Ciudad Autónoma de Buenos Aires y Paraná, Entre Ríos, Argentina. Se usó una modalidad virtual y otra presencial. Se contactó a los participantes por celulares, redes sociales o personalmente en escuelas o universidades. De este modo se pudo determinar si cumplían los criterios de inclusión de la muestra: tener de 12 a 40 años, residir en Paraná o Ciudad Autónoma de Buenos Aires, estar en contacto con alguno de los progenitores y poseer celular. El contacto mediante celulares o redes sociales — por ejemplo, con mails masivos - fue utilizado para invitar a participar en la investigación al adulto emergente o al joven adulto - mayor de edad — . En caso de que el participante manifestara estar interesado en colaborar, se acordaba un encuentro personal en el lugar que designara (su casa, un bar, la facultad, por ejemplo) o se enviaban por correo electrónico los cuestionarios mediante un link de Google Drive. El correo electrónico contenía una explicación del propósito de la investigación y de los requisitos para participar (tener entre 18 y 40 años, residir en Buenos Aires o Paraná, entre otros), un consentimiento informado para que se firmara digitalmente y el link con los tests. Por otra parte, el protocolo no se podía completar sin tildar una opción en la cual el participante aceptaba participar voluntariamente. Un $63 \%$ de la muestra respondió el protocolo de esta última manera. En el caso de la recolección presencial, se brindaba una explicación oral de la investigación y se firmaba un consentimiento escrito. El grupo de adolescentes $(20 \%)$ fue evaluado presencialmente en una escuela secundaria de Paraná y — previamentese solicitó el consentimiento de los progenitores o tutores mediante una nota en el cuaderno de comunicaciones de la escuela. Con este grupo, 
los datos se recolectaron en la escuela durante las horas que la institución destinó para este fin. Se escogió esta aproximación porque el contacto con un menor de edad mediante redes sociales o vía mail podría considerarse anti-ético, debido a que el menor habría podido completar el protocolo sin el consentimiento parental. Un 17\% de los adultos emergentes respondió presencialmente en la universidad a la cual están afiliados los autores, ya que se acercaron a dicha institución luego de ser contactados vía online, o se los contactó en cursos de la carrera de Psicología de la institución. Los instrumentos fueron aplicados por los autores del manuscrito y se tardó alrededor de 30 minutos en aplicarlos a quienes lo respondieron presencialmente. Se aseguró la confidencialidad y la participación voluntaria en todo el proceso de recolección de datos mediante un consentimiento informado escrito.

\section{Análisis de datos}

Los datos se analizaron en el programa SPSS 23.0 (IBM Corporation, 2015) para extraer estadísticos descriptivos (medias, desvíos, etc.) e inferenciales (coeficiente alfa de Cronbach para medir la consistencia interna y correlaciones de Pearson para evaluar la validez de constructo). Como el análisis de componentes principales se desaconseja (Lloret-Segura, Ferreres-Traver, Hernández-Baeza, \& Tomás-Marco, 2014), y como se deseaba explorar los factores que emergerían de la escala, se llevó a cabo un análisis paralelo (AP) con el programa Factor 8.10 (Lorenzo-Seva \& Ferrando, 2013). De esta manera, se usó un número de replicaciones igual a 100 y percentil de representación de simulaciones de .95. Para dicho análisis, se usó rotación promin debido a que se postulaba que los factores estarían asociados (Blanca \& Bendayan, 2018; Karadağ et al.,
2015). Asimismo, dicha rotación permite que los factores sean oblicuos, facilitando la simplificación de la estructura factorial (Lorenzo-Seva, 2013). Se usó el método ULS (cuadrados mínimos no ponderados) debido a la naturaleza ordinal de los datos y a que las opciones de respuesta eran solamente de cuatro alternativas.

Los valores de asimetría tenían una distribución que se apartaba ligeramente de la normalidad, ya que iban de .13 a 1.06, mientras que los de curtosis iban de .17 a 1.20, por lo cual se llevó a cabo un análisis factorial confirmatorio (AFC) con el método WLS (cuadrados mínimos ponderados) debido a que los ítems eran ordinales y contaban con menos de cinco opciones de respuesta (Brown, 2006; Lloret-Segura et al., 2014) y porque los datos se alejaban de la distribución normal (Byrne, 2012; Kline, 2015; Tabachnick \& Fidell, 2013). Se usó el programa MPLUS 6.0 (Muthén \& Muthén, 2011) para dicho análisis.

La muestra se dividió aleatoriamente en dos sub-muestras de tamaños casi idénticos. Con el primer grupo (500 participantes) se llevó a cabo el AP, mientras que con el grupo restante (552 participantes) se realizó el AFC. Se optó por este enfoque basado en los datos o bottom up - primero un análisis exploratorio y luego uno confirmatorio - porque se sabe que las estructuras factoriales de un instrumento pueden variar de un estudio a otro o cuando se está en un proceso de adaptación de un test (Fehm \& Hoyer, 2004; Wells \& Davies, 1994). Para evaluar el ajuste del modelo confirmatorio, se tuvieron en cuenta los índices CFI, TLI, RMSEA y SRMR. Valores de CFI y TLI por encima de .90 y RMSEA y SRMR por debajo de .10 fueron considerados adecuados (Bentler, 1992; Byrne, 2010), aunque existen criterios más exigentes que proponen CFI y TLI mayores que 95 y RMSEA y SRMR menores que .05 (Hu \& Bentler, 1999). En la actualidad, inclusive, se postulan criterios de CFI mayor que $.97 \mathrm{y}$ 
RSMEA y SRMR menores que 07 (Hair, Black, Babin, \& Anderson, 2010). Que el $\chi^{2}$ no sea significativo es un criterio muy exigente y sensible al tamaño de la muestra (Byrne, 2010), por lo cual no se tuvo en cuenta para evaluar el ajuste.

\section{Resultados}

Para evaluar la estructura factorial de la Escala de Phubbing, se llevó a cabo un AP con los 10 ítems. El cálculo del índice de adecuación muestral Kaiser-Meyer-Oklin $(\mathrm{KMO}=.83)$ y la prueba de esfericidad de Bartlett $\chi^{2}(18)=2702.40$ $p<.001$ dieron cuenta de la intercorrelación de los ítems y de la pertinencia del análisis.

Los resultados del análisis arrojaron la sugerencia de un modelo trifactorial. Dicha estructura explicaba un $61 \%$ de la varianza, todos los ítems presentaron cargas factoriales por encima de .38 en sus respectivas dimensiones y no se encontraron cargas factoriales complejas, como se muestra en la Tabla 1. El ajuste del modelo fue satisfactorio: $\chi_{(18)}^{2}=4633.75, \mathrm{CFI}=.99, \mathrm{TLI}=.98 \mathrm{y}$ RMSEA $=.03$. Los factores fueron denominados interferencia en la comunicación, obsesión con el celular y problemas psicosociales, y explicaban una varianza de 39\%, 12\% y 10\% respectivamente. Las correlaciones entre los factores fueron $r=$ $.51, .47$ y $.45 ; p<.01$, respectivamente.

Tabla 1.

Análisis paralelo de los ítems de la Escala de Phubbing

\begin{tabular}{lccc}
\hline Item & \multicolumn{3}{c}{ Factores } \\
\hline & Factor 1 & Factor 2 & Factor 3 \\
\hline 1. Cuando estoy con & .72 & \\
otras personas, empie- & & \\
zo a mirar de reojo a mi & & \\
celular. & & \\
\hline
\end{tabular}

2. Cuando estoy con mis amigos, siempre estoy ocupado con mi celular.

3. La gente se queja por el tiempo que paso con mi celular.

4. Cuando estoy con mi familia, siempre estoy ocupado con mi celular.

5. Creo que el estar ocupado con mi celular puede hacer enojar a mi mejor amigo o novio/a.

6. Mi celular está siempre cerca mío.

7. Cuando me despierto por la mañana, lo primero que hago es ver el celular.

8. Siento que me falta algo si no tengo mi celular.

9. El uso de mi celular 60 se incrementa día a día.

10. El tiempo dedicado a las actividades sociales, personales y escolares disminuye por el uso que hago del celular.

\begin{tabular}{llll}
\hline Varianza explicada & $39 \%$ & $12 \%$ & $10 \%$ \\
\hline Varianza explicada to- & & \\
tal $(61 \%)$ & & \\
\hline
\end{tabular}

Nota. Solo se muestran las cargas factoriales por encima de 30 .

Se puso a prueba dicho modelo con un AFC. En el Gráfico 1 se presenta el modelo a poner a prueba. Los resultados indicaron que el ajuste del mismo era muy aceptable, como se ve en la Tabla 
2. Se puso a prueba también un modelo bifactorial similar al postulado por los autores.

Como se ve en la Tabla 2, el modelo bifactorial presentó un ajuste menos adecuado que el de tres factores, ya que la diferencia $\Delta \chi^{2}$ era significativa: $p<.001$, mientras que la diferencia
$\Delta$ CFI era .021 , superando el valor $>.01$ señalado para ser significativo a este respecto (Byrne, 2012). Además, los valores de CFI, TLI, RMSEA y SRMR eran más satisfactorios para el modelo trifactorial.

Tabla 2

Ajustes para los modelos de la Escala de Phubbing.

\begin{tabular}{lccccccccccc}
\hline & $\boldsymbol{\chi}^{2}$ & $\boldsymbol{g} \boldsymbol{l}$ & $\boldsymbol{p}$ & $\mathbf{C F I}$ & TLI & RMSEA & SRMR & $\Delta \boldsymbol{\chi}^{2}$ & $\boldsymbol{p} \Delta \boldsymbol{\chi}^{2}$ & $\Delta \boldsymbol{g l}$ & $\Delta$ CFI \\
\hline $\begin{array}{l}\text { Modelo } \\
\text { trifacto- }\end{array}$ & 173.159 & 32 & $<.001$ & .969 & .958 & .079 & .073 & & & & \\
$\quad$ rial & & & & & & & & & & & \\
$\begin{array}{l}\text { Modelo } \\
\text { bifacto- }\end{array}$ & 275.332 & 34 & $<.001$ & .948 & .931 & .100 & .112 & 102.173 & .001 & 2 & .021 \\
$\quad$ \\
$\quad$
\end{tabular}

Nota. $g l=$ grados de libertad. $C F I=$ Comparative Fix Index. TLI $=$ Tucker-Lewis Index. RMSEA = raíz del residuo cuadrático promedio. $\mathrm{SRMR}=$ residuales estandarizados al cuadrado. $\Delta \chi^{2}=$ diferencia de $\chi^{2}$ entre los modelos. $\Delta g l=$ diferencia entre los grados de libertad de los modelos. $\Delta$ CFI diferencia de CFI entre los modelos.

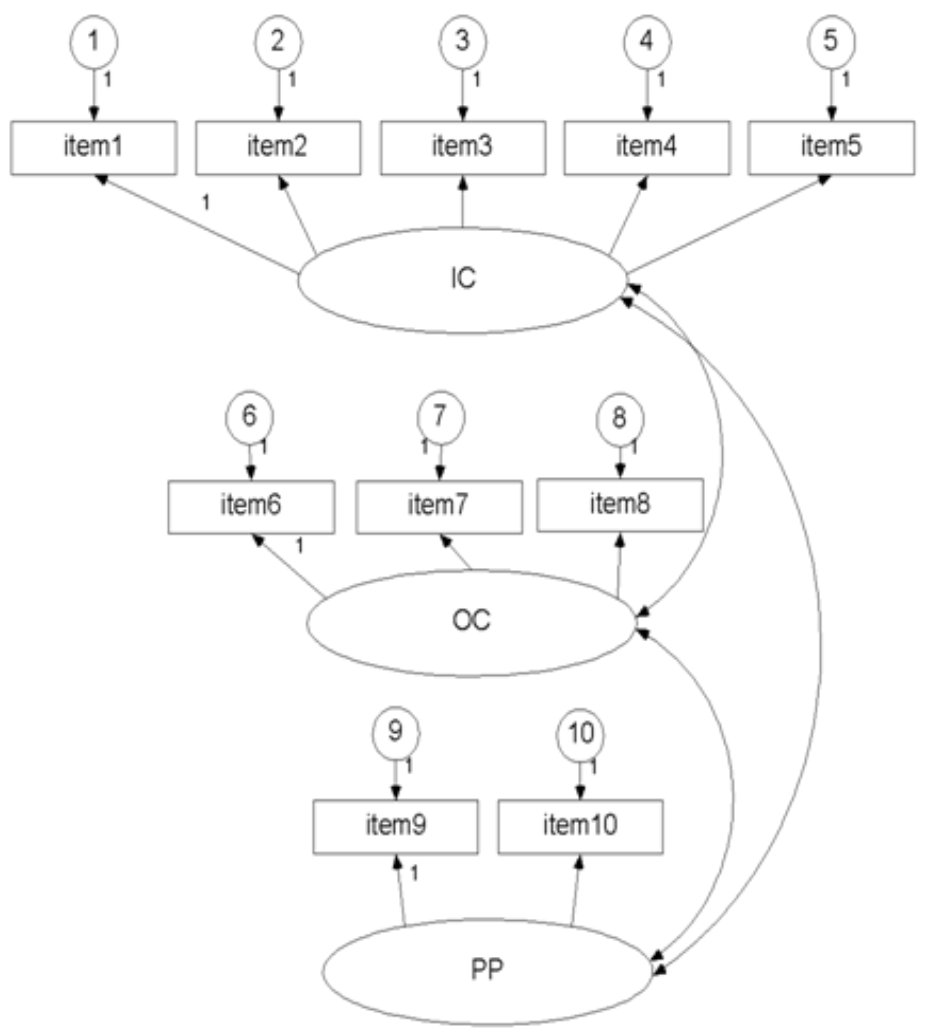

Gráfico 1. Modelo trifactorial de la Escala de Phubbing. 
Como se ve en la Tabla 2, el modelo bifactorial presentó un ajuste menos adecuado que el de tres factores, ya que la diferencia $\Delta \chi^{2}$ era significativa: $p<.001$, mientras que la diferencia $\Delta$ CFI era .021 , superando el valor $>.01$ señalado para ser significativo a este respecto (Byrne, 2012). Además, los valores de CFI, TLI, RMSEA y SRMR eran más satisfactorios para el modelo trifactorial.

Con respecto a la consistencia interna de la escala, se obtuvieron coeficientes alfa de Cron- bach con valores de .67 para interferencia en la comunicación; .66 para obsesión con el celular y .58 para problemas psicosociales. Como se muestra en la Tabla 3, la correlación de cada elemento con el total para cada dimensión se hallaba por encima de .38 para todos los ítems, excepto para el número cinco. El coeficiente alfa de Cronbach mejoró a .74 al eliminar la pregunta de su respectiva dimensión

Tabla 3

Consistencia interna de la Escala de Phubbing.

\begin{tabular}{|c|c|c|c|c|}
\hline Dimensión & $\begin{array}{l}\text { Media de escala } \\
\text { si el elemento se } \\
\text { ha suprimido }\end{array}$ & $\begin{array}{c}\text { Varianza de } \\
\text { escala si el } \\
\text { elemento se ha } \\
\text { suprimido }\end{array}$ & $\begin{array}{l}\text { Correlación } \\
\text { total de elemen- } \\
\text { tos corregida }\end{array}$ & $\begin{array}{l}\text { Alfa de Cron- } \\
\text { bach si el } \\
\text { elemento se ha } \\
\text { suprimido }\end{array}$ \\
\hline \multicolumn{5}{|l|}{ Interferencia con celular } \\
\hline $\begin{array}{l}\text { 1. Cuando estoy con otras personas, empie- } \\
\text { zo a mirar de reojo a mi celular. }\end{array}$ & 9.18 & 7.03 & .53 & .55 \\
\hline $\begin{array}{l}\text { 2. Cuando estoy con mis amigos, siempre } \\
\text { estoy ocupado con mi celular. }\end{array}$ & 9.86 & 7.44 & .54 & .55 \\
\hline $\begin{array}{l}\text { 3. La gente se queja por el tiempo que paso } \\
\text { con mi celular. }\end{array}$ & 10.15 & 7.08 & .46 & .58 \\
\hline $\begin{array}{l}\text { 4. Cuando estoy con mi familia, siempre es- } \\
\text { toy ocupado con mi celular. }\end{array}$ & 9.68 & 7.53 & .46 & .58 \\
\hline $\begin{array}{l}\text { 5. Creo que el estar ocupado con mi celu- } \\
\text { lar puede hacer enojar a mi mejor amigo o } \\
\text { novio/a. }\end{array}$ & 9.73 & 8.03 & .17 & .74 \\
\hline
\end{tabular}

Obsesión con celular

\begin{tabular}{lllll}
\hline 6. Mi celular esta siempre cerca mío. & 8.02 & 3.43 & .52 & .52
\end{tabular}

7. Cuando me despierto por la mañana, lo

8.05

3.08

.42

.61 primero que hago es ver el celular.

$\begin{array}{lll}8.54 & 2.39 & .49\end{array}$

.53 celular.

Problemas psicosociales

9. El uso de mi celular se incrementa día 2.58 1.67 .38 a día. 
Con respecto a la validez de constructo, se hallaron correlaciones significativas entre la edad y los puntajes en las tres dimensiones del phubbing. La edad se asoció con interferencia en la comunicación, obsesión con el celular y problemas psicosociales: $r=-.13,-.05,-.12$ y -.15 , respectivamente. Dichas asociaciones fueron significativas a un nivel de $p<.01$, con la excepción de una significación marginal $p<.09$ para la relación entre interferencia con la comunicación y edad.

Se llevaron a cabo correlaciones entre conflicto-antagonismo percibido con madres/padres y los puntajes en las dimensiones de phubbing. Como se ve en la Tabla 4, se hallaron correlaciones positivas entre las variables. Esto es, a mayor puntaje para cada una de las tres dimensiones del phubbing, mayor nivel de conflicto-antagonismo con ambos progenitores.

\section{Tabla 4}

Correlación entre la Escala de Phubbing y conflicto-antagonismo con progenitores.

\begin{tabular}{ccc}
\hline & CA Madre & CA Padre \\
\hline IC & $.20^{* *}$ & $.22^{* *}$ \\
PP & $.11^{* *}$ & $.14^{* *}$ \\
OC & $.15^{* *}$ & $.18^{* *}$ \\
\hline
\end{tabular}

Nota. ${ }^{*} p<.01$. IC: interferencia con celular. PP: problemas psicosociales. OC: obsesión con el celular. CA: conflicto-antagonismo.

\section{Discusión}

El propósito del presente trabajo fue evaluar las propiedades psicométricas de la Escala de
Phubbing de Karadağ et al. (2015), la cual es una de las pocas disponibles en el mundo para medir dicha conducta. A pesar de la frecuencia del uso del celular en la Argentina y de su relevancia psicosocial, no existen estudios que hayan examinado las propiedades psicométricas de un cuestionario de phubbing en nuestra región, ni tampoco en América Latina. Por lo cual, la fortaleza de este trabajo estuvo en ser el primero de la región en evaluar las propiedades psicométricas de dicha escala. Por otra parte, se empleó una muestra de tamaño grande: se constituyó una muestra intencional de 1245 participantes de la Ciudad Autónoma de Buenos Aires y Paraná, Argentina, con edades de 15 a 40 años (70\% de mujeres). Los participantes contestaron la Escala de Karadağ et al. (2015), la subescala Conflicto-Antagonismo con progenitores (padres y madres) del Inventario Red de Relaciones de Furman y Buhrmester (1992) y un cuestionario demográfico.

En lo referente a la estructura factorial, tanto en el AP como en el AFC, se halló un modelo con tres factores. En el análisis paralelo, dicha estructura explicaba un $61 \%$ de la varianza y todos los ítems obtuvieron cargas por encima de .38 en sus respectivos factores, sin que se presentaran ítems complejos. Los factores fueron denominados interferencia en la comunicación, obsesión con el celular y problemas psicosociales. En lo relativo al AFC, este obtuvo un ajuste muy adecuado para dicha estructura, ya que los valores de CFI y TLI se hallaron por encima de .90 y el RMSEA y SRMR estuvo por debajo de .10 (Bentler, 1992; Byrne, 2010). Más aún, los valores cumplieron con los criterios más exigentes que requieren CFI 
y TLI mayores que .95 y RMSEA y SRMR estaban bastante cercanos al requerimiento de valores menores que 05 (Hu \& Bentler, 1999). Incluso se aproximaron a los criterios más actuales que requieren CFI mayor que 97 y RSMEA y SRMR menores que .07 (Hair et al., 2010). Por otra parte, el modelo mencionado mostró un ajuste más adecuado - como lo indicaba la diferencia de $\Delta \chi^{2}$ y $\triangle$ CFI y valores más adecuados de CFI, TLI, RMSEA y SRMR - que el modelo bifactorial propuesto por los autores (Karadağ et al., 2015), también replicado en España (Blanca \& Bendayan, 2018). La dimensión interferencia en la comunicación, no obstante, se compuso de los mismos ítems que en el modelo de Karadağ et al. (2015). La diferencia en el modelo aquí detectado puede deberse a las diferencias sociales, culturales y económicas entre la Argentina y dichos países. Sin embargo, mayor investigación es necesaria respecto a este punto.

En relación con su consistencia interna, el coeficiente alfa de Cronbach fue de .67 para interferencia en la comunicación, .66 para obsesión con el celular y .58 para problemas psicosociales. Al eliminar la pregunta cinco por su baja consistencia, el índice aumentó a .74. Por lo tanto, los valores pueden ser considerados adecuados para interferencia en la comunicación, ya que un índice entre .70 y .80 se postula como una estimación de consistencia interna satisfactoria (DeVellis, 2012; Kaplan \& Saccuzzo, 2006). Las otras dos dimensiones, en cambio, presentaron una consistencia interna menos satisfactoria. No obstante, puede ser aceptable un criterio más laxo — por ejemplo, valores alrededor de .60- para escalas que cuentan con un escaso número de ítems (Loewenthal, 2001). La subescala obsesión con el celular quedó conformada por tres ítems, mientras que problemas psicosociales quedó compuesta por solo dos ítems. En España, Blanca y Bendayan (2018) detectaron valores más elevados de consistencia para interferencia en la comunicación y obsesión con el celular: 85 y .76, respectivamente. También en Turquía se hallaron valores similares: 87 y .85, respectivamente (Karadağ et al., 2015). Sin embargo, dichas subescalas tenían mayor cantidad de ítems debido a la estructura de dos factores. Por otra parte, se debería evaluar si la baja consistencia se debe a que las dimensiones quedaron conformadas por menor cantidad de ítems o a otros problemas - como la administración online- en quienes lo contestaron virtualmente. Por ejemplo, en este último formato no está disponible el investigador para evacuar dudas con respecto a los ítems o a otros aspectos de la encuesta. Si bien se comprobó que la administración presencial versus online presenta escasas diferencias al tomar los recaudos necesarios, algunas facetas de la recolección online pueden ser cuestionables (Miller, Crowe, Weiss, Maples-Keller, \& Lynam, 2017; Weigold, Weigold, \& Russell, 2013). Futuros estudios deberían examinar cómo funciona psicométricamente el test aplicado presencialmente versus el test online. Se podría estudiar, también, su invariancia de medición de acuerdo al formato.

Con respecto a su validez concurrente, se observaron asociaciones significativas entre las tres dimensiones del phubbing y la edad. Se detectó que, a menor edad, mayor nivel de phubbing. Dichas asociaciones fueron estadísticamente significativas, con la excepción de la asociación con interferencia en la comunicación, que resultó marginalmente significativa. Dicha asociación es esperable, ya que la gente joven, como los adolescentes y adultos emergentes, se ve atraída hacia los celulares y redes sociales debido al período de la vida en el que se encuentra. Tanto en la adolescencia como en la adultez emergente, las exploraciones de la identidad — como las amorosas - se vuelven centrales, aunque en la tercera década de la vida dicha exploración es más focalizada (Ar- 
nett, 2013). Como señalan las investigaciones, dichos grupos usan más intensamente los celulares con el fin de entrar en contacto con sus pares - como amigos o posibles parejas amorosas - o para desarrollar su identidad, gracias a la posibilidad de conectarse en todo momento o lugar (Karacic \& Oreskovic, 2017; Zboralski et al., 2009). Por otra parte, el anonimato y desinhibición que permiten las TIC también generan gran atracción en los jóvenes a la hora de explorar su identidad o para la experimentación sexual y amorosa.

Asimismo, se detectaron asociaciones de las tres dimensiones del phubbing con el conflicto-antagonismo percibido con padres y madres, lo cual es una nueva prueba de la validez concurrente de la escala. Cabe destacar que el tamaño de las correlaciones resultó pequeño o mediano, aunque las mismas eran estadísticamente significativas. Por otra parte, que una conducta como el phubbing - incluso en adolescentes tardíos, adultos emergentes y adultos jóvenes - se asocie con la calidad de las relaciones con padres y madres, pone de manifiesto el importante valor de la familia para el ajuste psicosocial. Además, la asociación del phubbing con las interacciones negativas percibidas fue de un tamaño similar para ambos progenitores. Muchos estudios señalan que en la Argentina los valores familiares son mucho más altos que en los países del primer mundo, como los Estados Unidos o Europa del norte (Facio \& Resett, 2012), lo cual podría explicar los presentes resultados. No obstante, un estudio en los Estados Unidos - aunque con adolescentes - también detectó que los problemas en las relaciones entre hijos adolescentes y sus progenitores - debido al uso excesivo de las nuevas tecnologíasse asociaban con mayor sintomatología depresiva y conductas de cyberbullying en sus hijos (Stockdale et al., 2018). Como señalan Karadağ et al. (2016), el phubbing puede generar muchos problemas psicosociales, desde afectar las relaciones interpersonales hasta dañar las habilidades para la comunicación, generando por ejemplo dificultades para el contacto visual en las comunicaciones cara a cara o interpretaciones erróneas en las mismas.

Que las asociaciones halladas en el presente estudio fuesen pequeñas o medianas puede explicarse por el hecho de que los constructos en psicología se hallan multideterminados. Como señalan Karadağ et al. (2015), el phubbing es el resultante de muchos factores, así como las adicciones a las nuevas tecnologías. Además de la edad y la calidad de las relaciones con progenitores, otros factores pueden asociarse al phubbing: por ejemplo, estilos de afrontamiento, impulsividad, problemas emocionales, control inhibitorio, nivel socioeconómico, entre otros.

A pesar de las diferencias sociales y culturales de la Argentina con Turquía y España, estos hallazgos sugerirían que este instrumento presenta evidencias de bondad psicométrica en una muestra argentina, aunque con una estructura de tres factores.

Este estudio tiene una serie de limitaciones que deben ser mencionadas: Una de ellas es la de haber sido llevado a cabo con una muestra intencional. Asimismo, la muestra se compuso de mayor cantidad de mujeres que de varones, $y$ está comprobado que el género puede introducir diferencias en el uso de TIC, aunque esto no ha sido demostrado aún para el phubbing. Por otra parte, los datos fueron recogidos mediante el autoinforme, lo cual podría inflar artificialmente las relaciones entre las variables por la varianza compartida en el método de recolección de datos (Richardson, Simmering, \& Sturman, 2009). Asimismo, los instrumentos de autoinforme - como las escalas - tienen conocidas limitaciones: sesgo en las respuestas, falta de honestidad en las respuestas, marcar alternativas socialmente deseables o extremas. Además, un porcentaje de la muestra 
contestó de manera online. Que el estudio haya sido transversal no permite evaluar la confiabilidad test-retest de la escala, ni tampoco permite inferir la direccionalidad de la causalidad entre las variables. Es posible que el phubbing genere mayores conflictos con los progenitores a causa de los reclamos y reproches de los padres por el uso excesivo del celular; pero también es posible que aquellos sujetos que perciben una peor calidad en sus relaciones con padres y madres se refugien en las nuevas tecnologías con el fin de sobrellevar el malestar psicológico. Asimismo, es posible que una tercera variable - como rasgos de personalidad, problemas emocionales o estilos de afrontamiento - haga covariar al phubbing y la percepción de las relaciones parentales. Por otra parte, la validez de constructo se evaluó con las interacciones negativas con progenitores, por lo cual algunos sujetos quedaron excluidos. Finalmente, la validez concurrente se exploró con correlaciones de Pearson y no con otros métodos más sofisticados, como los modelos estructurales.

Futuros estudios deberían examinar esta problemática en muestras de mayor tamaño y seleccionadas al azar de diversas provincias de la Argentina, para poder generalizar los resultados. Una cuestión relevante es que futuras investigaciones deberían ser longitudinales, tanto para examinar su consistencia test-retest como para determinar qué tan estable temporalmente es el phubbing — considerando los rápidos cambios que se dan con los celulares y sus aplicaciones-. Además, futuros estudios deberían evaluar la escala con la teoría de la respuesta al ítem. Sería deseable evaluar la validez concurrente con modelos estructurales y con otras conductas disfuncionales asociadas con las nuevas tecnologías, como la adicción a las redes sociales, el fear of missing out, el sexting, el cyberstalking, entre otras. Por último, se debería examinar el papel que juegan otras variables psicosociales, como la personalidad o los problemas emocionales.

\section{Referencias}

Abramova, O., Baumann, A., Krasnova, H., \& Lessmann, S. (junio, 2017). To phub or not to phub: Understanding off-task smartphone usage and its consequences in the academic environment. Trabajo presentado en la $25^{\circ}$ European Conference on Information Systems (ECIS), Guimarães, Portugal. Recuperado de https:// aisel.aisnet.org

Arnett, J. J. (2013). Adolescence and Emerging Adulthood. A cultural approach. Nueva Jersey, NJ: Pearson.

Bentler, P. M. (1992). On the fit of models to covariances and methodology to the Bulletin. Psychological Bulletin, 112(3), 400-404. doi: 10.1037/0033-2909.112.3.400

Blanca, M. J., \& Bendayan, R. (2018). Spanish version of the Phubbing Scale: Internet addiction, Facebook intrusion, and fear of missing out as correlates. Psicothema, 30(4), 449-454. Recuperado de http://www. psicothema.com

Brown, T. A. (2006). Confirmatory factor analysis for applied research. Nueva York, NY: Guilford.

Byrne, B. M. (2010). Multivariate Applications Series. Structural Equation Modelling with AMOS: Basic Concepts, Applications, and Programming ( $2^{\mathrm{a}}$ ed.). Nueva York, NY: Routledge.

Byrne, B. M. (2012). Multivariate Applications Series. Structural equation modelling with MPLUS: Basic concepts, applications, and programming. Nueva York, NY: Routledge.

Capilla-Garrido, E., \& Cubo-Delgado, S. (2017). Phubbing. Conectados a la red y desconectados de la realidad. Un análisis en relación al bienestar psicológico. Píxel-Bit. Revista de Medios y Educación, 50, 173185. doi: 10.12795/pixelbit.2017.i50.12

Chotpitayasunondh, V., \& Douglas, K. M. (2016). How "phubbing" becomes the norm: The antecedents and 
consequences of snubbing via smartphone. Computers in Human Behavior, 63, 9-18. doi: 10.1016/j. chb.2016.05.018

DeVellis, R. F. (2012). Scale Development: Theory and Applications ( $3^{\mathrm{a}}$ ed.). Thousand Oaks, CA: Sage.

Facio, A., \& Resett, S. (2012). Argentina. En J. J. Arnett (Ed.), Adolescent Psychology Around the World (pp. 151-162). Nueva York, NY: Psychology. doi: $10.4324 / 9780203809129$

Facio, A., \& Resett, S. (2014). Work, romantic relationships and life satisfaction in Argentinean emerging adults. Emerging Adulthood, 2(1), 27-35. doi: $10.1177 / 2167696813515854$

Facio, A., Resett, S., Micocci, F., \& Mistrorigo, C. (2007). Emerging adulthood in Argentina: An age of diversity and possibilities. Child Development Perspectives, 1(2), 115-118. doi: 10.1111/j.17508606.2007.00025.x

Facio, A., Resett, S., Mistrorigo, C., \& Micocci, F. (2006). Adolescentes argentinos. Cómo piensan y sienten. Buenos Aires, Argentina: Lugar.

Fehm, L., \& Hoyer, J. (2004). Measuring thought control strategies: The Thought Control Questionnaire and a look beyond. Cognitive Therapy and Research, 28(1), 105-117. doi: 10.1023/b:cotr.0000016933.41653.dc

Furman, W., \& Buhrmester, D. (1992). Age and sex differences in perceptions of networks of personal relationships. Child Development, 63(1), 103-115. doi: $10.2307 / 1130905$

Hair, J. F., Black, W. C., Babin, B. J., \& Anderson, R. E. (2010). Multivariate Data Analysis. Nueva York, NY: Pearson.

Hartung, C. M., Little, C. S., Allen, E. K., \& Page, M. C. (2011). A psychometric comparison of two self-report measures of bullying and victimization: Differences by sex and grade. School Mental Health, 3(1), 44-57. doi: 10.1007/s12310-010-9046-1

Hiscock, D. (2004). Cell phones in class: This, too, shall pass? Community College Week, 16, 4-5.

Hong, F.-Y., Chiu, S.-I., \& Huang, D.-H. (2012). A model of the relationship between psychological charac- teristics, mobile phone addiction and use of mobile phones by Taiwanese university female students. Computers in Human Behavior, 28(6), 2152-2159. doi: 10.1016/j.chb.2012.06.020

Hu, L.-T., \& Bentler, P. M. (1999). Cutoff criteria for fit indexes in covariance structure analysis: Conventional criteria versus new alternatives. Structural Equation Modelling. A Multidisciplinary Journal, 6(1), 1-55. doi: 10.1080/10705519909540118

Huang, S., Hu, Y., Ni, Q., Qin, Y., \& Lü, W. (2019). Parent-children relationship and internet addiction of adolescents: The mediating role of self-concept. Current Psychology, 2(3), 1-9. doi: 10.1007/s12144019-00199-9

IBM Corporation. (2015). IBM SPSS Statistics for Windows (23.0). [Software de cómputo]. Armonk: IBM Corporation.

Instituto Nacional de Estadísticas y Censos. (2019). Encuesta permanente de hogares 2018. Recuperado de https://www.indec.gob.ar

International Telecommunication Union. (2013). Measuring the Information Society. Ginebra, Suiza: International Telecommunication Union.

International Test Commission. (2017). The ITC Guidelines for Translating and Adapting Tests ( $2^{\mathrm{a}}$ ed.). Recuperado de https://www.intestcom.org

Kaplan, R. M., \& Saccuzzo, D. P. (2006). Pruebas psicológicas: Principios, aplicaciones y temas ( $6^{\mathrm{a}}$ ed.). Madrid, España: International Thomson.

Karacic, S., \& Oreskovic, S. (2017). Internet addiction through the phase of adolescence: A questionnaire study. JMIR Mental Health,4(2), 11-16. doi: 10.2196/ mental.5537

Karadağ, E., Tosuntaş, Ş. B., Erzen, E., Duru, P., Bostan, N., Şahin, B. M. ... Babadağ, B. (2015). Determinants of phubbing, which is the sum of many virtual addictions: A structural equation model. Journal of Behavioral Addictions,4(2), 60-74. doi: 10.1556/2006.4.2015.005

Karadağ, E., Tosuntaş, Ş. B., Erzen, E., Duru, P., Bostan, N., Şahin, B. M. ... Babadağ, B. (2016). The virtual 
world's current addiction: Phubbing. Addicta: The Turkish Jounal on Addictions, 3(2), 250-269. doi: 10.15805/addicta.2016.3.0013

Kline, R. B. (2015). Principles and practice of structural equation modelling ( $4^{\mathrm{a}}$ ed.). Nueva York, NY: Guilford.

Lenhart, A. (2015). Teens, social media and technology overview 2015. Washington, D. C.: Pew Research Center. Recuperado de https://www.pewresearch. org/internet/2015/04/09/introduction-teens-tech

Liu, R.-D., Wang, J., Gu, D., Ding, Y., Oei, T. P., Hong, W. ... Li, Y.-M. (2019). The effect of parental phubbing on teenager's mobile phone dependency behaviors: The mediation role of subjective norm and dependency intention. Psychology Research and Behavior Management, 12, 1059-1069. doi: 10.2147/PRBM. S224133

Lloret-Segura, S., Ferreres-Traver, A., Hernández-Baeza, A., \& Tomás-Marco, I. (2014). El análisis factorial exploratorio de los ítems: Una guía práctica, revisada y actualizada. Anales de Psicología, 30(3), 11511169. doi: 10.6018/analesps.30.3.199361

Loewenthal, K. M. (2001). An introduction to psychological tests and scales ( $2^{\mathrm{a}}$ ed.). East Sussex, UK: Psychology.

Lorenzo-Seva, U. (2013). Why rotate my data using Promin? Technical Report. Tarragona, España: Department of Psychology, Universitat Rovira i Virgili.

Lorenzo-Seva, U., \& Ferrando, P. J. (2013). Manual of the Program Factor v. 8.10. Tarragona, España: Department of Psychology, Universitat Rovira i Virgili. Recuperado de http://www.psicologia.urv.cat/ca

Miller, J. D., Crowe, M., Weiss, B., Maples-Keller, J. L., \& Lynam, D. R. (2017). Using online, crowdsourcing platforms for data collection in personality disorder research: The example of Amazon's Mechanical Turk. Personality Disorders: Theory, Research, and Treatment, 8(1), 26-34. doi: 10.1037/per0000191

Muñiz, J., Elosua, P., \& Hambleton, R. K. (2013). Directrices para la traducción y adaptación de los test: Se- gunda edición. Psicothema, 25(2), 151-157. Recuperado de http://www.psicothema.com

Muthén, L., \& Muthén, B. (2011). Mplus User's Guide. Los Angeles, CA: Muthén \& Muthén.

Nazir, T., \& Pişkin, M. (2016). Phubbing: A technological invasion, which connected the world but disconnected humans. The International Journal of Indian Psychology, 3(4), 39-46. Recuperado de https://ijip.in

Resett, S., \& González-Caino, P. C. (2019). Propiedades psicométricas de un Cuestionario de Trolling en una muestra argentina. Revista Argentina de Ciencias del Comportamiento, 11(1), 48-57. doi: 10.32348/1852.4206.v11.n1.20870

Richardson, H. A., Simmering, M. J., \& Sturman, M. C. (2009). A tale of three perspectives: Examining post hoc statistical techniques for detection and correction of common method variance. Organizational Research Methods, 12(4), 762-800. doi: 10.1177/1094428109332834

Sánchez-Rosas, J. (2015). The Achievement Emotions Questionnaire-Argentine (AEQ-AR): Internal and external validity, reliability, gender differences and norm-referenced interpretation of test scores. Revista Evaluar, 15(1), 41-74. Recuperado de https://revistas.unc.edu.ar/index.php/revaluar

Solís-Salazar, M. (2015). The dilemma of combining positive and negative items in scales. Psicothema, 27(2), 192-200. Recuperado de http://www.psicothema. com

Stockdale, L. A., Coyne, S. M., \& Padilla-Walker, L. M. (2018). Parent and child technoference and socioemotional behavioral outcomes: A nationally representative study of 10- to 20-year-old adolescents. Computers in Human Behavior, 88, 219-226. doi: 10.1016/j.chb.2018.06.034

Suárez-Álvarez, J., Pedrosa, I., Lozano, L. M., García-Cueto, E., Cuesta, M., \& Muñiz, J. (2018). Using reversed items in Likert scales: A questionable practice. Psicothema, 30(2), 149-158. Recuperado de http:// www.psicothema.com 
Tabachnick, B. G., \& Fidell, L. S. (2013). Using multivariate statistics ( $6^{\mathrm{a}}$ ed.). Boston, MA: Allyn \& Bacon/ Pearson Education.

Ugur, N. G., \& Koc, T. (2015). Time for digital detox: Misuse of mobile technology and phubbing. Procedia - Social and Behavioral Sciences, 195, 1022-1031. doi: 10.1016/j.sbspro.2015.06.491

Van Sonderen, E., Sanderman, R., \& Coyne, J. C. (2013). Ineffectiveness of reverse wording of questionnaire items: Let's learn from cows in the rain. PLOS ONE, 8(7), 1-7. doi: 10.1371/journal.pone.0068967

Vanden-Abeele, M. M. P., Antheunis, M. L., \& Schouten, A. P. (2016). The effect of mobile messaging during a conversation on impression formation and interaction quality. Computers in Human Behavior, 62, 562569. doi: 10.1016/j.chb.2016.04.005

Walsh, S. P., White, K. M., Hyde, M. K., \& Watson, B. (2008). Dialling and driving: Factors influencing intentions to use a mobile phone while driving. Accident Analysis and Prevention, 40(6), 1893-1900. doi: 10.1016/j.aap.2008.07.005

Weigold, A., Weigold, I. K., \& Russell, E. J. (2013). Examination of the equivalence of self-report survey-based paper-and-pencil and internet data collection methods. Psychological Methods, 18(1), 53-70. doi: $10.1037 / \mathrm{a} 0031607$

Wells, A., \& Davies, M. I. (1994). The Thought Control Questionnaire: A measure of individual differences in the control of unwanted thoughts. Behaviour Research and Therapy, 32(8), 871-878. doi: 10.1016/0005-7967(94)90168-6

Zboralski, K., Orzechowska, A., Talarowska, M., Darmosz, A., Janiak, A., Janiak, M. ... Gałecki, P. (2009). The prevalence of computer and Internet addiction among pupils. Postępy Higieny i Medycyny Doświadczalnej, 63, 8-12. Recuperado de http://www.phmd.pl 